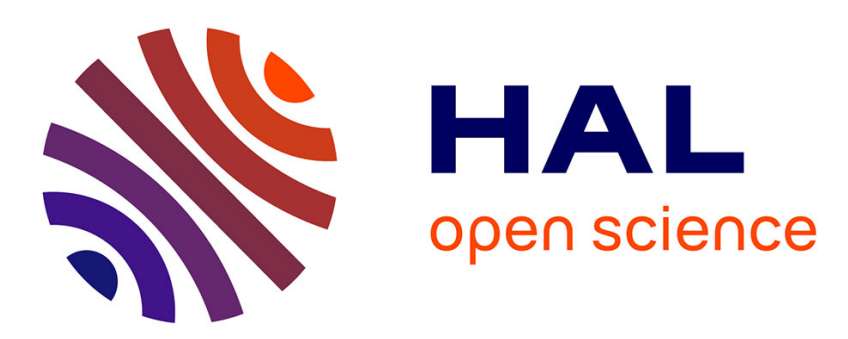

\title{
Ion acoustic waves and double-layers in electronegative expanding plasmas
}

\author{
Nicolas Plihon, Pascal Chabert
}

\section{To cite this version:}

Nicolas Plihon, Pascal Chabert. Ion acoustic waves and double-layers in electronegative expanding plasmas. Physics of Plasmas, 2011, 18, pp.082102. 10.1063/1.3620405 . hal-01113303

\section{HAL Id: hal-01113303 https://hal.science/hal-01113303}

Submitted on 4 Feb 2015

HAL is a multi-disciplinary open access archive for the deposit and dissemination of scientific research documents, whether they are published or not. The documents may come from teaching and research institutions in France or abroad, or from public or private research centers.
L'archive ouverte pluridisciplinaire HAL, est destinée au dépôt et à la diffusion de documents scientifiques de niveau recherche, publiés ou non, émanant des établissements d'enseignement et de recherche français ou étrangers, des laboratoires publics ou privés. 


\title{
Ion acoustic waves and double-layers in electronegative expanding plasmas
}

\author{
Nicolas Plihon ${ }^{1, a)}$ and Pascal Chabert ${ }^{2}$ \\ ${ }^{1}$ Laboratoire de Physique Laboratoire de Physique, École Normale Supérieure de Lyon, CNRS, Université de \\ Lyon, 46 allée d'Italie, 69364 Lyon Cedex, France \\ ${ }^{2}$ Laboratoire de Physique des Plasmas, École Polytechnique, UPMC, Universite Paris Sud-11, CNRS, \\ 91128 Palaiseau Cedex, France
}

(Received 9 May 2011; accepted 11 July 2011; published online 8 August 2011)

\begin{abstract}
Ion acoustic waves and double-layers are observed in expanding plasmas in electronegative gases, i.e., plasmas containing an appreciable fraction of negative ions. The reported experiments are performed in argon gas with a variable amount of $\mathrm{SF}_{6}$. When varying the amount of $\mathrm{SF}_{6}$, the negative ion fraction increases and three main regimes were identified previously: (i) the plasma smoothly expands at low negative ion fraction, (ii) a static double-layer (associated with an abrupt potential drop and ion acceleration) forms at intermediate negative ion fraction, (iii) double-layers periodically form and propagate (in the plasma expansion direction) at high negative ion fraction. In this paper, we show that transition phases exist in between these regimes, where fluctuations are observed. These fluctuations are unstable slow ion acoustic waves, propagating in the direction opposite to the plasma expansion. These fluctuations are excited by the most unstable eigenmodes and display turbulent features. It is suggested that the static double layer forms when the ion acoustic fluctuations become non-linearly unstable: the double layer regime being a bifurcated state of the smoothly expanding regime. For the highest negative ion fraction, a coexistence of (upstream propagating) slow ion acoustic fluctuations and (downstream) propagating double layers was observed. (C) 2011 American Institute of Physics. [doi:10.1063/1.3620405]
\end{abstract}

\section{INTRODUCTION}

Low pressure electronegative plasmas, i.e., plasmas containing an appreciable amount of negative ions, have been intensively studied in the last decades, not only because they are used in many industrial applications but also because their fundamental properties differ significantly from the more usual electropositive plasmas. ${ }^{1}$ Among these peculiar properties, temporal instabilities of the plasma parameters have been observed in several conditions: for example, in capacitive discharges, ${ }^{2}$ at capacitive/inductive mode transitions in inductive discharges, ${ }^{3,4}$ or transport instabilities in expanding plasmas. The first report of such transport instabilities, where the plasma is produced in a source chamber and expands in a larger chamber, was given by Tuszewski and coworkers ${ }^{5,6}$ who measured ion saturation current fluctuation on a probe located in the expansion region of an inductive discharge driven at $0.46 \mathrm{MHz}$ and working in $\mathrm{Ar} / \mathrm{SF}_{6}$ gas mixtures. Using both a fluid model and a kinetic model, Tuszewski and Gary have theoretically shown that, in an electronegative plasma, the counter streaming of oppositely charged ions (positive and negative ions) is linearly unstable when the difference in streaming velocities exceeds a threshold. ${ }^{7}$ They proposed that the fluctuations observed are the result of this counter-streaming ion instability. It was, however, argued later that the fluctuations of the plasma parameters could be due to periodic downstream propagating double-layers, ${ }^{8}$ as was observed by Plihon and coworkers in the expansion chamber of an $13.56 \mathrm{MHz}$ inductive discharge in $\mathrm{Ar} / \mathrm{SF}_{6}$ gas mixtures. It was also shown that double layers

\footnotetext{
${ }^{\text {a)} E l e c t r o n i c ~ m a i l: ~ n i c o l a s . p l i h o n @ e n s-l y o n . f r . ~}$
}

are a ubiquitous phenomenon in expanding electronegative plasmas: they could be stationary ${ }^{9,10}$ or propagative ${ }^{8}$ and develop in various electronegative gas mixture. ${ }^{10,11}$ Slow ion acoustic waves were also observed both without the double layer and on top of the double layer, but this phenomenon was only presented in Plihon Ph.D. thesis. ${ }^{12}$ It is the purpose of this paper to present in details the link between ion acoustic waves and double layer formation and propagation. Besides these experimental investigations and their theoretical interpretation, similar plasma conditions were observed in a number of numerical simulations. Meige and coworkers observed double layers in expanding electronegative plasmas using particles in cells (PIC) simulations. ${ }^{13}$ They observed both static double layers, at moderate negative ion fraction, and (upstream) propagating double layer, at high negative ion fraction. Using PIC simulations, Kawamura and coworkers also observed static double layers in expanding plasmas, associated with ion acoustic waves in a narrower range of parameters. ${ }^{14} \mathrm{~A}$ detailed analysis of the ion acoustic waves $^{15}$ showed similar features with the experimental observations of Tuszweski and coworkers ${ }^{5,7}$ and Plihon. ${ }^{12}$ Moreover, the model introduced by Tuszewski and Gary, and extended to include collisions in Ref. 15, showed a very good agreement with the PIC simulations.

In this paper, the link between ion acoustic waves and double layers, based on the analysis of experimental data obtained in expanding electronegative plasma, will be addressed and compared with previous theoretical and numerical investigations. The paper is organized as follows. The experimental arrangement is described in Sec. II. In Sec. III, the conditions for double-layer formation, discussed in previous publications, are summarized as a background of 
what lies ahead. The ion acoustic waves are analyzed in Sec. IV. In particular, the turbulent nature of the ion acoustic fluctuations is emphasized, and their influence on the overall dynamics is discussed. Conclusions are given in a last dedicated section.

\section{EXPERIMENTAL APPARATUS}

The experimental setup is shown schematically in Figure 1. The plasma vessel is sketched in the left-hand panel and consists of a source chamber above a $32 \mathrm{~cm}$ diameter expansion chamber. The source chamber is a $15 \mathrm{~cm}$ diameter, $30 \mathrm{~cm}$ long, and $0.9 \mathrm{~cm}$ thick Pyrex cylinder surrounded by a double saddle field type helicon antenna. ${ }^{16}$ The fancooled antenna is powered through a close-coupled L-type matching network by an rf power supply operating at 13.56 $\mathrm{MHz}$ and capable of delivering up to $2 \mathrm{~kW}$ forward power. The time-averaged input power was recorded as the difference between the time-averaged forward and reflected powers. The Pyrex cylinder is housed in a $20 \mathrm{~cm}$ diameter and $30 \mathrm{~cm}$ long grounded aluminum cylinder. A grid attached to the other end of the source tube confines the plasma and isolates it from a turbo-molecular pump that routinely maintains base pressures of $10^{-6}$ mbar. The discharge was run in $\mathrm{Ar} / \mathrm{SF}_{6}$ gas mixtures and $\mathrm{Ar} / \mathrm{O}_{2}$ gas mixtures. Most of the results presented in the present paper have been obtained in $\mathrm{Ar} / \mathrm{SF}_{6}$ gas mixtures. The partial gas pressures of $\mathrm{Ar}$ and $\mathrm{SF}_{6}$ were determined by controlling the flows and the total pressure was measured using a Baratron pressure gauge.

Measurements reported here were made along the revolution axis ( $z$ axis) of the experimental set-up. Plasma parameters in the steady-state regimes are determined using a passively compensated Langmuir probe, ${ }^{17}$ having a $0.25 \mathrm{~mm}$ diameter and $6 \mathrm{~mm}$ long platinum wire tip. Time-averaged plasma potential $V_{p}$, electron density $n_{e}$ and electron temperature $T_{e}$ were deduced from the I-V characteristics of the cylindrical probe using a SMARTSOFT data acquisition system ${ }^{18}$
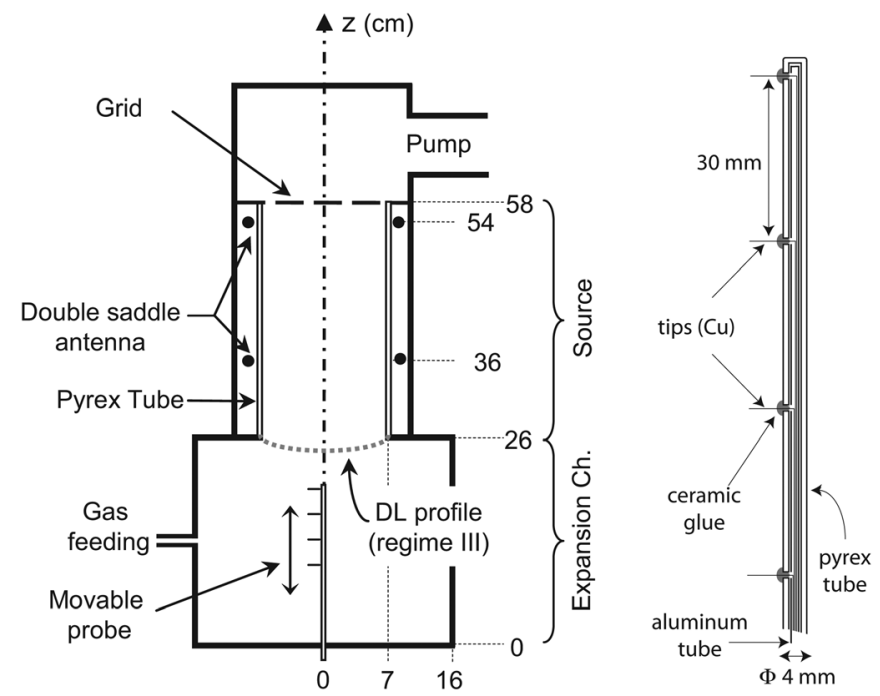

FIG. 1. Schematic of the experimental setup and the probe used to simultaneously probe the positive ion saturation current at four position on axis. Each of the probe tips is independently biased at $-36 \mathrm{~V}$. A typical profile of the static double layer is displayed on top of the experimental setup. or from usual I-V curve processing. ${ }^{19}$ In the unstable regimes, the plasma parameter fluctuations are synchronously probed, giving access to time-resolved spatio-temporal evolution of the plasma parameters, as described in Ref. 8. The propagative fluctuations are analyzed from simultaneous acquisition of the positive ion saturation current $I_{\text {sat }}$ of a quadruple tipped uncompensated copper probe, sketched in the right-hand panel in Figure 1. The probe was built as thin as possible: it is composed of an outer $4 \mathrm{~mm}$ diameter Pyrex tube, surrounding an inner aluminum grounded tube to avoid capacitive coupling at the driving $13.56 \mathrm{MHz}$ radiofrequency. The tips are $4 \mathrm{~mm}$ long and $0.25 \mathrm{~mm}$ in diameter and are equally spaced by $30 \mathrm{~mm}$. The probes are dc biased at $-36 \mathrm{~V}$ by small individual batteries and temporal evolution of the positive ion flux at four positions on axis are simultaneously digitized on a $200 \mathrm{MHz}$ digital oscilloscope with 8 bits resolution.

Measurement of the negative ion density $n_{\text {- }}$ can be achieved either by the electrostatic probe measurements ${ }^{20}$ or with the probe based laser induced photodetachment (LIP) technique, ${ }^{21}$ which rely on the measurement of the increase of the electron current on a probe due to electrons release when photons with sufficient energy interact with negative ions. Here, LIP measurements were performed using a tripled Nd:YAG beam (355 nm photons). Although two independent negative ion density measurements are available, the absolute measurement of the negative ion fraction $\alpha=n_{-} / n_{e}$ is not easily obtained: the electrostatic probe method is limited to large negative ion fraction regimes (and is very sensitive to the unknown negative ion temperature $T_{-}$), while $355 \mathrm{~nm}$ photons may not photodetach all fluorine containing negative ions. Based on the discussion of the experimental procedure and its limitations in Ref. 11, the negative ion fraction values discussed in this paper are corrected experimental values (i.e., twice the value measured from laser photodetachment and one third of the value measured from electrostatic probes).

The purpose of this paper is to study the features of the plasma equilibria and their transitions when increasing the negative ion fraction $\alpha$. While the negative ion fraction $\alpha$ is usually the physical parameter controlling the plasma equilibrium, it is not a straightforward experimental control parameter: our experimentally adjustable parameter being the relative concentration of electronegative gases in the gas mixture. All results were thus obtained as a function of the relative $\mathrm{SF}_{6}$ concentration $C_{\mathrm{SF}_{6}}$ or the relative $\mathrm{O}_{2}$ concentration $\mathrm{Co}_{2}$ but will be analyzed with the negative ion fraction $\alpha$ as being the control parameter. The typical operational conditions are $1 \mathrm{mT}$ Torr plasma, maintained by $600 \mathrm{~W}$ forward electrical power. In the source region, typical electron density is $n_{e} \sim 10^{17} \mathrm{~m}^{-1}$ and electron temperature $T_{e} \sim 3 \mathrm{eV}$. Negative ion fractions up to ten were obtained in $\mathrm{Ar} / \mathrm{SF}_{6}$ mixtures, while remaining below one in $\mathrm{Ar} / \mathrm{O}_{2}$ mixtures.

\section{DOUBLE LAYER FORMATION IN ELECTRONEGATIVE PLASMAS}

In this section, the conditions for the formation of double layers in expanding electronegative plasmas, and their 
main features will be briefly recalled. By expanding plasma, we mean that the power is deposited to the plasma in a finite (source) region, and that the plasma freely expands in a larger chamber. As detailed in Refs. 8, 9, and 11, three important regimes were identified when increasing the negative ion fraction:

- at low negative ion fraction: a steady-state plasma continuously expands from the source region into the expansion chamber

- at intermediate negative ion fraction: a steady-state, static, double-layer forms at the interface between the source and the expansion chambers

- at high negative ion fraction: double layers periodically form at the interface between the source and the expansion chambers and propagate in the expansion chamber.

These regimes will now be illustrated by showing, in Figure 2, the spatial evolution of the plasma potential measured in $1 \mathrm{mTorr}$ plasma, for increasing $\mathrm{SF}_{6}$ concentrations. A typical plasma potential evolution at low $\mathrm{SF}_{6}$ concentration $(4 \%)$ is shown in Figure 2(a) as black plain symbols. The plasma is generated in the source region $(26 \mathrm{~cm}<z<60 \mathrm{~cm})$ and continuously expands. A smooth plasma potential gradient is observed from $30 \mathrm{~V}$ in the source region to $15 \mathrm{~V}$ at the end of the expansion chamber. The negative ion fraction is null in the source region, while it is peaked at the top of the expansion chamber $\left(\alpha_{\max }=0.6\right.$ for $C_{\mathrm{SF}_{6}}=4 \%$ ). In this regime, no plasma parameter fluctuations have been observed. In this manuscript, this regime will be referred to as "Regime I," for steady state plasma equilibrium, where no double layer is present.

For $\mathrm{SF}_{6}$ concentrations between 8 and $13 \%$, a static double layer forms at the interface between the source and the expansion chambers. A typical plasma potential evolution in this regime is shown in Figure 2(a) as open symbols. A steep gradient of the plasma potential is observed at the sourceexpansion chamber interface (note that the plasma potential is unchanged in the source region as compared to the previous regime). It was shown in Ref. 8 and 11 that a moderate amplitude double layer $\left(\Phi_{\mathrm{DL}} \sim 2 T_{e}\right)$ was created. The double layer (D.L.) is an internal sheath between an electropositive plasma upstream-in the source-and an electronegative plasma downstream-in the expansion chamber. The negative ion fraction is null in the source; it is peaked in the downstream region at the D.L. location $\left(\alpha_{\max }=3\right.$ for $C_{\mathrm{SF}_{6}}=8 \%$ ). Once again, for intermediate values of the negative ion fraction, no plasma parameter fluctuations have been observed in the presence of the static double layer. In this manuscript, this regime will be referred to as "Regime III," where a static double layer forms at the interface between the source and the expansion chamber.

For higher $\mathrm{SF}_{6}$ concentrations, the double layer destabilizes and becomes periodically propagating. Double layers form at the source/expansion chamber interface and propagate downstream. A typical spatio-temporal profile of the plasma potential is shown in Figure 2(b), normalized to the period $T$, which lies in the ms range. The amplitude of the propagating double layer is similar to that of the static double layer. Once again, the source plasma is negative-ion free,

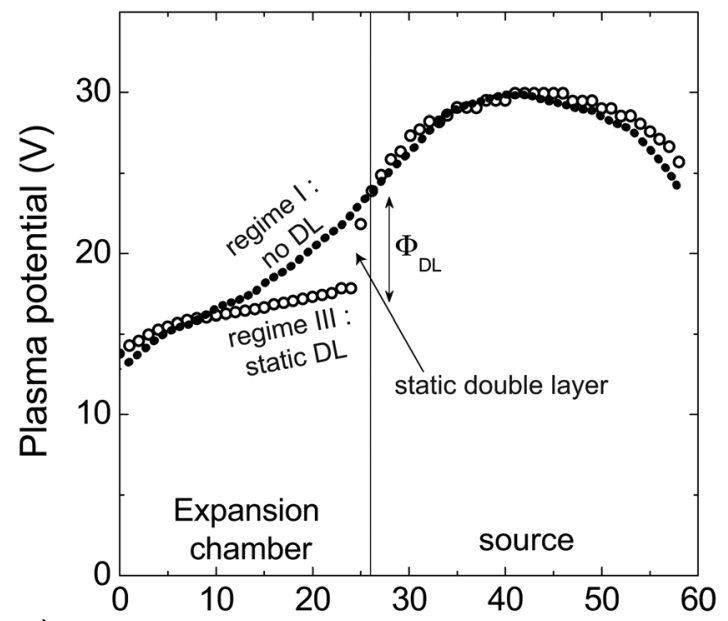

a) $\mathrm{Z}(\mathrm{cm})$

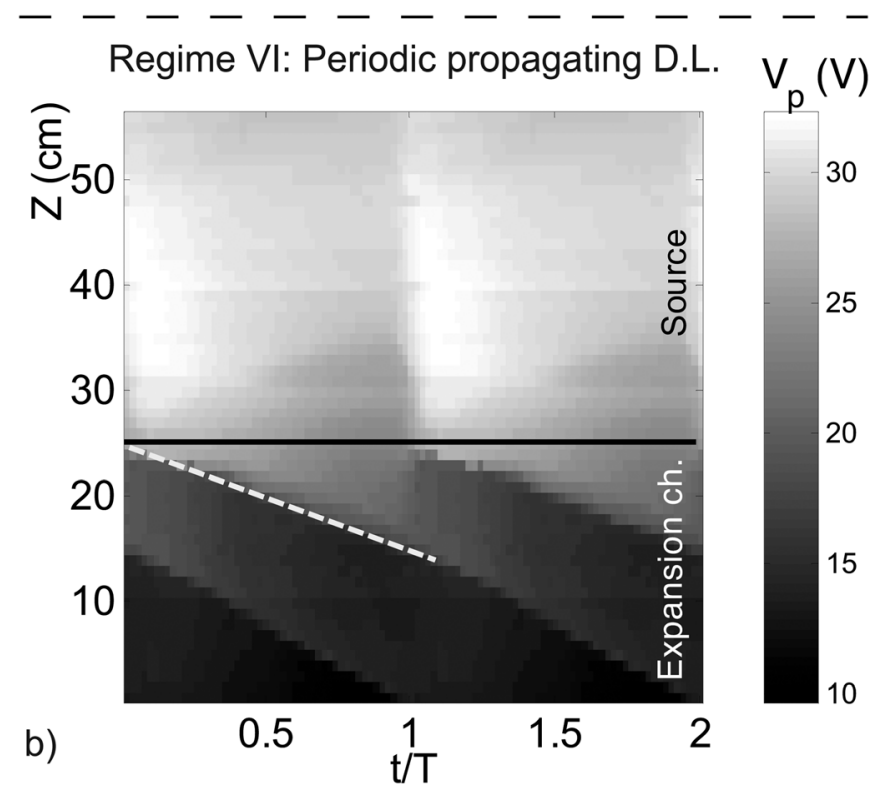

FIG. 2. (a) Axial evolution of the plasma potential for a (0.96:0.04) Ar:SF6 mixture (plain symbols) and a (0.91:0.9) mixture (open symbol). The plain symbols situation is the continuously expanding plasma (Regime I) while the open symbols situation shows a static double-layer at $\mathrm{z}=24 \mathrm{~cm}$ (Regime III). (b) Spatio-temporal evolution of the plasma potential on axis for a (0.75:0.25) Ar:SF 6 mixture, normalized to the period of propagation ( $T=1.3 \mathrm{~ms}$ ), showing the downstream propagation of double layers (Regime VI). Regimes obtained at $1 \mathrm{mT}$ Torr, $600 \mathrm{~W}$.

while the negative ion fraction downstream of the propagating D.L may reach 10 . In this manuscript, this regime will be referred to as "Regime VI."

\section{TRANSITIONS AND ION ACOUSTIC FLUCTUATIONS}

\section{A. General features}

The scenario described in section III was simplified, and reality is indeed more complex. Figure 3 shows samples of the temporal evolution of the absolute value of the ion saturation current, in the expansion chamber, for increasing values of the $\mathrm{SF}_{6}$ concentration. A quick examination of Figure 3 shows the two time-independent regimes already described: Regime I (smoothly expanding plasma) and Regime III (static double layer, where the ion plasma density is 


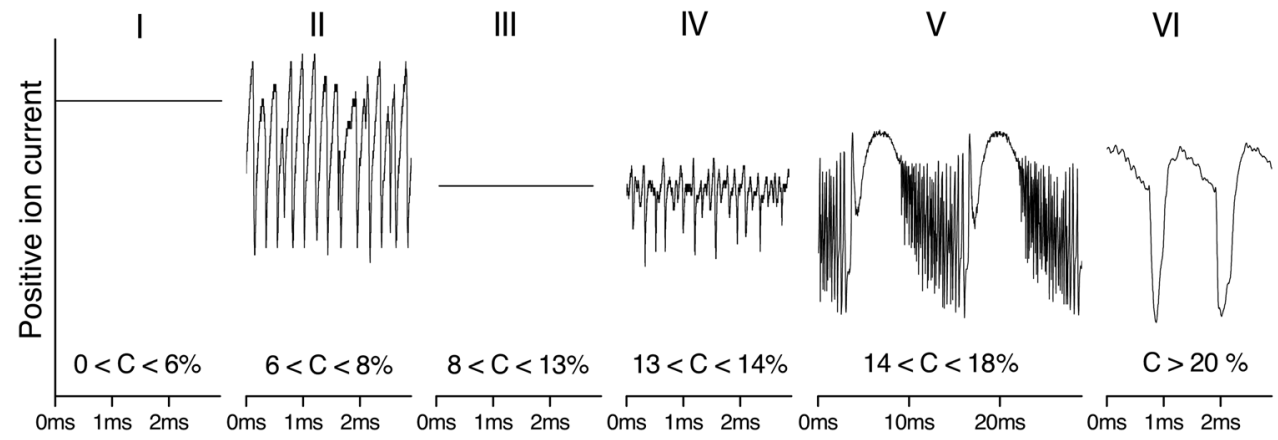

FIG. 3. Schematic temporal evolution of the positive ion saturation current for increasing values of the $\mathrm{SF}_{6}$ concentration $\mathrm{C}$ from the continuously expanding regime to the periodically propagating double layer regime (refer to the text for a precise description). These schematic traces are observed at $z \approx 20 \mathrm{~cm}$.

decreased, downstream of the D.L, as compared with Regime I). The periodically downstream propagating double layer, i.e., Regime VI described above, is also depicted, for the largest $\mathrm{SF}_{6}$ concentrations. Moreover, three time-dependent and fairly aperiodic transition regimes (labeled II, IV, and V) are also displayed; we will show in the following that the observed fluctuations are slow ion-acoustic waves. The features of these three transitional regimes are briefly described here, before being detailed in the dedicated subsections:

- Regime II. On top of a time-averaged continuouslyexpanding plasma, ion acoustic waves are driven unstable in the expansion chamber. These waves are propagating upstream, and their amplitude increases with the negative ion fraction. We will show in the following subsection that these fluctuations have turbulent-like features.

- Regime IV. On top of the static double layer, ion acoustic waves are driven unstable in the downstream region of the double layer. These fluctuations also propagate upstream. This regime may only be observed transiently when transiting to Regime V.

- Regime V. Regime where ion acoustic waves are driven unstable on top of a smoothly expanding plasma. Slow plasma parameters evolution drives the ion acoustic waves non-linearly unstable, leading to the formation of a downstream propagating double-layer.

The detailed operational conditions for the transition between these regimes obviously depend on the gases used, the plasma density, and the operating pressure. In mixtures with highly electronegative gases, such as $\mathrm{SF}_{6}$, the six regimes were observed, whereas in $\mathrm{Ar} / \mathrm{O}_{2}$ mixtures, only the first three regimes were observed. ${ }^{11}$ For instance in $\mathrm{Ar} / \mathrm{O}_{2}$ mixtures, Regime $\mathrm{I}$ is observed for $\mathrm{O}_{2}$ concentrations $0<\mathrm{Co}_{2}<68 \%$, Regime II is observed for $68 \%<\mathrm{Co}_{2}$ $<72 \%$, and Regime III is observed for $\mathrm{Co}_{2}>72 \%$.

A detailed characterization of the ion acoustic fluctuations of Regimes II, IV, and V is provided in the remaining of the manuscript, and the link between these ion acoustic fluctuations and double layer formation is discussed.

\section{B. Characteristics of ion acoustic turbulence}

In this subsection, the characteristics of the fluctuations observed in Regime II are studied in details. We will show later that the fluctuations observed in Regime IV share the same features. Typical simultaneous time series of ion saturation current from the 4-tip probe are shown in Figure 4(a). The first observation is that the fluctuations clearly show a dominant frequency and could be considered as pseudo-harmonic waves at frequency $f_{\max }$. The second observation is that the fluctuations coherently propagate upstream, as shown by the propagation of the two patterns circled in Figure 4(a) at $t \sim 0.6 \mathrm{~ms}$ and $t \sim 0.75 \mathrm{~ms}$. The last observation from these time traces is that the amplitude of the fluctuations increases when propagating upstream in the expansion chamber; this will be shown more clearly in Figure 5(b). Note that, due to the very rapid growth rates of the linear instability, the linearly unstable regime, where waves are excited from vanishingly small noise, could not be observed
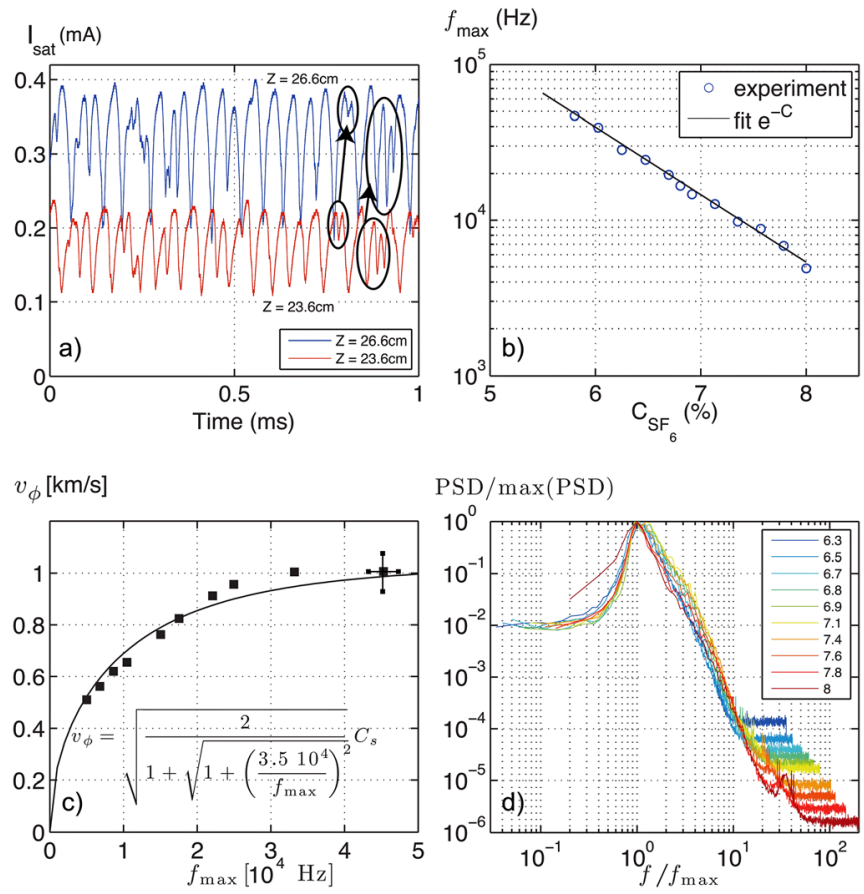

FIG. 4. (Color online) Characterization of Regime II. (a) Typical temporal evolution of the positive ion saturation current for two locations of the probe. (b) Evolution of the most probable harmonic of the ion current fluctuations as a function of the $\mathrm{SF}_{6}$ concentration. (c) Experimental (dots) and theoretical dispersion relation for the single-harmonic like fluctuations obtained when varying the $\mathrm{SF}_{6}$ concentration-relative error bars are shown for one experimental point. (d) Normalized power spectral densities (PSD) of the ion saturation current for increasing values of the $\mathrm{SF}_{6}$ concentration (blue to red). 

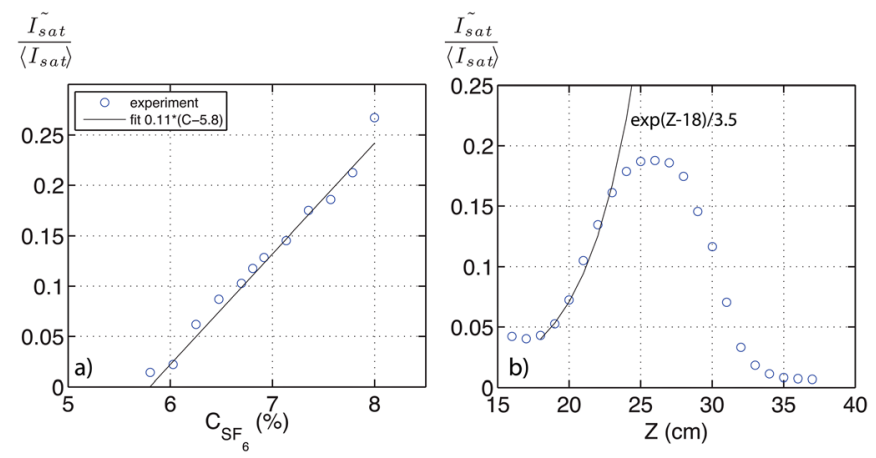

FIG. 5. (Color online) (a) Evolution of the maximum normalized standard deviation of the ion acoustic waves as a function of the $\mathrm{SF}_{6}$ concentration in Regime II. (b) Typical spatial evolution of the normalized standard deviation of the ion acoustic wave ((0.924:0.076) Ar:SF 6 mixture).

and probed accurately. All features described here are thus features of the non-linearly saturated state.

The time series in Figure 4(a) were obtained for a fairly high value of the negative ion fraction in Regime II, but the observed fluctuations show similar behaviors for all negative ion fraction values in Regime II. However, the dominant frequency of the waves strongly depends on the value of the negative ion fraction, as shown in Figure 4(b). The value of $f_{\max }$ decreases exponentially when increasing the $\mathrm{SF}_{6}$ concentration. It is thus experimentally easy to modify the dominant frequency of the waves by changing the $\mathrm{SF}_{6}$ concentration, i.e., the negative ion fraction of the plasma, and to determine a dispersion relation for the pseudo-harmonic waves. Thanks to the 4-tip probe, the phase velocity is measured as $v_{\Phi}=\Delta z / \Delta t$, where $\Delta z=z_{2}-z_{1}$ is the distance between two probe tips at locations $z_{1}$ and $z_{2}$, respectively, and $\Delta t$ is the time lag at which the correlation between $I_{\text {sat }}\left(t, z=z_{1}\right)$ and $I_{\text {sat }}\left(t, z=z_{2}\right)$ is maximum. The experimental dispersion relation is shown in Figure 4(c), where the highest frequency corresponds to the lowest negative ion fraction. The most probable frequency is determined with a $\pm 5 \%$ uncertainty, and the phase velocity is computed with an estimated $7 \%$ uncertainty. A theoretical fit of the dispersion relation for ion acoustic modes, given by the following expression: ${ }^{22}$

$$
v_{\phi}(f)=\sqrt{\frac{2}{1+\sqrt{1+\left(\frac{f_{\text {collision }}}{f}\right)^{2}}}} C_{s},
$$

is also displayed in Figure 4(c). In Eq. (1), $f_{\text {collision }}$ is the ionneutral collision frequency and $C_{S}$ is the ion acoustic speed, which has to be modified for electronegative plasmas and reads

$$
C_{s}=\sqrt{\frac{k T_{e}}{M_{+}} \frac{1+\alpha}{1+\gamma \alpha}}
$$

where $M_{+}$is the mass of the dominant ions, and $\gamma=T_{e} / T_{-}$is the ratio of the negatively charged species temperatures. Since the frequencies of these pseudo-harmonic waves are moderate, ion-neutral collisions have to be taken into account when modeling the dispersion relation. The best fitting parameters in Eq. (1) are $f_{\text {collision }}=3.5 \times 10^{4} \mathrm{~Hz}$ and $C_{s}=1050 \mathrm{~m} \cdot \mathrm{s}^{-1}$. The actual ion-neutral collision frequency was estimated to lie around $f_{\text {collision }}=3 \times 10^{4} \mathrm{~Hz}$ in Ref. 7, from a typical $10^{-18} \mathrm{~m}^{2}$ cross section, ${ }^{1}$ in good agreement with our fit. The plasma parameters determined in Regime II ( $T_{e}=3 \mathrm{eV}, \alpha \sim 0.6$, and $\gamma \sim 15, M_{+}=40 \mathrm{amu}$ ) (Ref. 11) give a value of $C_{s}=1070 \mathrm{~m} . \mathrm{s}^{-1}$, again in very good agreement with the fit. From Figures 4(a)-4(c) and the above analysis, the fluctuations observed in Regime II are thus slow ion acoustic waves propagating upstream.

Up to now, only the pseudo-harmonic features of the ion acoustic fluctuations have been described. However, as seen in Figure 4(a), the fluctuations are not harmonic and are observed at all temporal scales. The normalized power spectral density (PSD) of the fluctuations is displayed in Figure $4(d)$ as a function of the frequency normalized to the most probable frequency $f_{\max }$. The normalized PSD collapse to a single master curve, which is a typical signature of turbulent signals. The PSD show that the energy of the fluctuations is injected at a given time-scale, or frequency $f_{\max }$, and cascade self-similarly toward larger frequencies. The probe measurements are not sufficiently resolved to show the dissipative time-scale of the turbulent cascade, but show an inertial-like cascade over 1 to 1.5 decade. The spectral index in the inertial range is rather steep: a $f^{-4}$ can be ascribed to the PSD of the ion saturation current.

As the negative ion fraction is increased, besides, the decrease of the value of the most unstable eigenvalue already shown, the amplitude of the saturated fluctuations increases. Figure 5(a) displays the evolution of the standard deviation of the ion saturation current $\widetilde{I}_{\mathrm{sat}}$ as a function of the $\mathrm{SF}_{6}$ concentration, again in Regime II. For $\mathrm{SF}_{6}$ concentrations above $8 \%$, a static double layer forms (to enter Regime III) and the conditions for slow ion acoustic wave instability vanish. This suggests that the static double layer is formed when the ion acoustic wave become non-linearly unstable: the double layer regime being a bifurcated state of the smoothly expanding regime.

A typical spatial evolution of the normalized ion acoustic wave amplitude is given in Figure 5(b). Ion acoustic waves are driven linearly unstable in the expansion chamber, and propagate upstream, while the amplitude of the waves increases. Data are compatible with an exponential spatial growth of the wave, with a spatial growth rate in the range [2-5] $\mathrm{cm}$ as shown in 5(b). The waves are kept non-linearly stable via some stabilizing processes such as damping by ion-neutral collisions, and their amplitude displays a plateau at the top of the expansion chamber. When entering deeper in the source region, the plasma becomes more and more electropositive and the ion acoustic waves are quickly damped (ion acoustic waves are indeed severely damped in electropositive plasmas and are usually not observed).

From the above observations, a plausible scenario is the following:

- Slow ion acoustic waves are driven linearly unstable in the expansion chamber, where the negative ion fraction is the highest. The most unstable eigenvalue being linked to the plasma negative ion fraction, as shown in Figure 4(b). 
- The most unstable ion acoustic wave grows exponentially in the expansion chamber while propagating upstream, displays a non-linearly saturated region, before being damped when entering the electropositive source plasma.

- The upstream propagating unstable ion acoustic waves non-linearly interact and give birth to a turbulent cascade, as shown in Figure 4(d).

- The amplitude of the ion acoustic fluctuations increases linearly with the $\mathrm{SF}_{6}$ concentration. For high enough negative ion fractions, the ion acoustic fluctuations are driven non-linearly unstable and a double layer forms.

Our experimental observations will now be compared with previous theoretical and numerical calculations of the stability conditions for ion acoustic waves in similar plasma conditions. Using a fluid model, Tuszewski and Gary showed that slow ion acoustic waves could be driven unstable when the (opposite) streaming velocities of negative ions and positive ions exceed a threshold. ${ }^{7}$ From the plasma potential evolution shown in Figure 2(a), negative ions are drifting in the upstream direction, while positive ions are drifting in the downstream direction, and for sufficient negative ion fraction, the instability threshold is met. The direction of propagation of the unstable ion acoustic waves depends on the balance of drifting and thermal velocities. In our experimental setup, they were observed to propagate in the upstream direction. Following the work of Tuszewski and Gary, Kawamura, and coworkers extended a kinetic model to include collisions. ${ }^{15}$ They found that the most unstable slow ion acoustic waves had pulsations in the $\left[10^{5}, 10^{6}\right]$ rad.s ${ }^{-1}$ range and wave vector in the $[100,1000] \mathrm{m}^{-1}$ range. From the experimental data presented in this manuscript, the characteristics of the pseudo-harmonic ion acoustic waves $\left(\omega \sim 10^{5}\right.$ rad.s $\mathrm{s}^{-1}$ and $\left.k \sim 10^{2} \mathrm{~m}^{-1}\right)$ are consistent with these previous theoretical calculations.

\section{Ion acoustic turbulence and double layers}

\section{Unstable ion acoustic fluctuations downstream of a static double layer}

Ion acoustic fluctuations were also observed on top of the static double layer regime: this is Regime IV previously mentioned. These fluctuations share the same characteristics as the one depicted for Regime II. Regime IV is very difficult to probe since it is stable in a very narrow range of parameters. It is likely that the physical control parameters very slowly evolve (while keeping all experimental control parameters constant) and that Regime IV is always quickly driven unstable. Regime IV was, thus, only transiently probed along the transition from Regime III (static double layer) to Regime V (very slowly propagating double layers). Figure 6(a) displays the time-averaged ion saturation current and its standard deviation as a function of axial position (since Regime IV is only observed transiently, no precise axial scan have been measured; only two locations of the 4-tip probe were scanned). The time averaged evolution of the ion saturation current shows the existence of the static double layer between 26 and $29 \mathrm{~cm}$. Figure 6(a) shows that

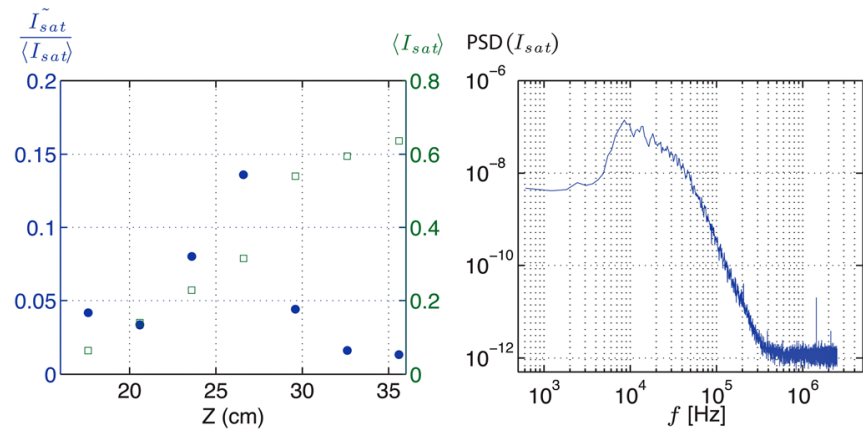

FIG. 6. (Color online) Regime IV: (a) Spatial evolution of the positive ion saturation current in regime IV: normalized amplitude of the fluctuations (full symbols), and time-averaged ion saturation current (open symbols). The right-hand side scale displays the value of the time averaged current and shows the existence of a double layer between axial position 26 and $29 \mathrm{~cm}$. The left hand side scale displays the normalized value of the standard deviation of the current. (b) PSD of ion acoustic fluctuations at location $26 \mathrm{~cm}$.

the ion acoustic fluctuations are driven unstable downstream of the double layer (in an electronegative plasma) and are quickly damped upstream of the double layer, in an electropositive plasma. All characteristics previously detailed for the fluctuations of regime II are recovered here. A typical PSD of the fluctuations, displayed in Figure 6(b), shows a dominant frequency $f_{\max } \sim 8 \mathrm{kHz}$, and the same shape for the PSD as a function of frequency. Moreover, similar time series (not shown) as well as similar values of the phase velocity $\left(850 \mathrm{~m} . \mathrm{s}^{-1}\right)$ have been observed, which confirm that the characteristics of the fluctuations of Regime IV are similar to those of Regime II.

We note here that Regime IV is very similar to the regime observed by Kawamura and coworkers: ${ }^{14,15}$ slow ion acoustic waves are observed downstream of a static double layer, and propagate upstream, before being damped at the double layer location. Indeed, in presence of a static double layer, positive ions beams were identified, ${ }^{11}$ and the fluid and kinetic models conditions for ion acoustic wave instability $^{7,14,15}$ could be met. It is important to note here that Kawamura and coworkers did not observe ion acoustic fluctuations without a double layer, i.e., Regime II was not observed in the numerical simulations. Kawamura and coworkers thus found that ion acoustic waves are driven unstable in a very narrow range of control parameters (narrower than the range for which a double layer forms), while we found the opposite conditions. This difference may be linked to details of the plasma equilibrium (gradients in plasma potential, collisional processes, and ion masses), modifying the stability criteria for slow ion acoustic waves.

\section{Ion acoustic fluctuations and propagating double layers}

For most of the negative ion fraction values above the static double layer regime, both ion acoustic fluctuations and propagating double layer coexist; this is Regime V. Typical time series of ion saturation current for seven axial positions are given in Figure 7; this figure has been reconstructed from scans with the 4-tip probe for two locations of the probe (i.e., 


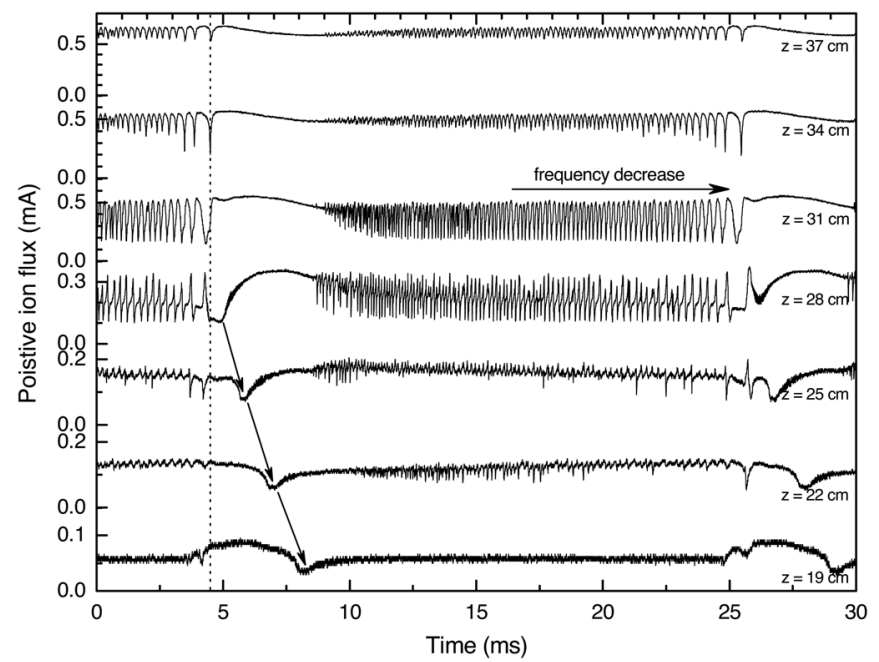

FIG. 7. Regime V. Ion saturation current time-series for a $(0.84: 0.16)$ Ar: $\mathrm{SF}_{6} \%$ mixture. See text for details.

only four of the time-series were acquired simultaneously). These time-series show the coexistence of upstream propagating slow ion acoustic waves and (very slowly) downstream propagating double layers; this will be discussed in this section. We start the description of the dynamics at time $t=4.5$ ms (i.e., at the location of the dashed line in Figure 7):

- A double layer forms at $t=4.5 \mathrm{~ms}$, between $z=28 \mathrm{~cm}$ and $z=31 \mathrm{~cm}$ (at the end of the source Pyrex tube): the ion saturation current suddenly increases at the double layer position; it is higher upstream, where the plasma density is higher.

- The double layer propagates downstream very slowly. This propagation is sketched by black arrows on the bottom traces in Figure 7: the propagation velocity is of the order of $30 \mathrm{~m} . \mathrm{s}^{-1}$. The downstream propagation is observed on all time traces for $z<28 \mathrm{~cm}$. It is also seen by examining the $z=28 \mathrm{~cm}$ time trace (for instance) for which at times $t>5 \mathrm{~ms}$ the ion saturation current increases, indicating that the double layer is subsequently below the probe location.

- A smoothly expanding plasma is recovered in the source and half of the expansion chamber at times $t>8 \mathrm{~ms}$. At times $t>8.5 \mathrm{~ms}$, the plasma conditions are such that slow ion acoustic waves are unstable at locations around the source/expansion chamber interface. Indeed, ion acoustic fluctuations are observed at $t>8.5 \mathrm{~ms}$. As for Regimes II and IV, these ion acoustic fluctuations propagate in the upstream direction, i.e., oppositely to the double layers. Note here that these ion acoustic fluctuations develop on a mean plasma profile very similar to the one of Regimes I and II.

- A very slow increase of the negative ion fraction is observed for times $9 \mathrm{~ms}<t<25 \mathrm{~ms}$. This slow negative ion fraction increase is inferred from the slow evolution of the ion acoustic fluctuations characteristics (decrease of ion acoustic pseudo-harmonic frequency and increase of the ion acoustic fluctuation amplitude) assuming that the ion acoustic fluctuations behave similarly as in Regime II. The time-scale for the slow evolution of the plasma param- eters is of the order of $10 \mathrm{~ms}$ for the operational conditions of Figure 7.

- When the negative ion fraction value is high enough, the ion acoustic waves are driven non-linearly unstable and a double layer forms at time $t=26 \mathrm{~ms}$.

The diversity of time-scales observed in the temporal evolution of the ion saturation current in Figure 7 is only observed for a very narrow range of parameters. The evolution of the time-series with the $\mathrm{SF}_{6}$ concentration is given in Figure 8 . When slightly increasing the $\mathrm{SF}_{6}$ concentration, the time during which ion acoustic fluctuations are observed is dramatically reduced. We argue here that the duration over which ion acoustic fluctuations are observed is linked to the (slow) time-scale dynamics of the negative ions. For a $16 \%$ $\mathrm{SF}_{6}$ concentration, the negative-ion time scale is a few tens of milliseconds: the electronegativity very slowly increases as compared with the ion acoustic fluctuation time scale. Tens of pseudo-periods are observed before the slow ion acoustic waves are driven non-linearly unstable (and as a result a double layer forms). For a $17 \% \mathrm{SF}_{6}$ concentration, the negative-ion time scale reduces to a few milliseconds: only a few pseudo-periods of slow ion acoustic waves are observed before these slow ion acoustic waves are driven non-linearly unstable. Above $21 \% \mathrm{SF}_{6}$ concentration, the negative-ion time scale is shorter than a pseudo period of the most (linearly) unstable ion acoustic wave: the wave is driven non-linearly unstable within less than half a period. In this regime, ion acoustic fluctuations are not easily observed. The difference between Regime V and Regime VI is thus the following: in Regime V, several pseudo-periods of ion acoustic fluctuations are observed between two consecutive double layer formations, while in Regime VI, no ion acoustic periodic fluctuations are observed. In Regime VI, the negative ion evolution time scale (linked to the non-linear

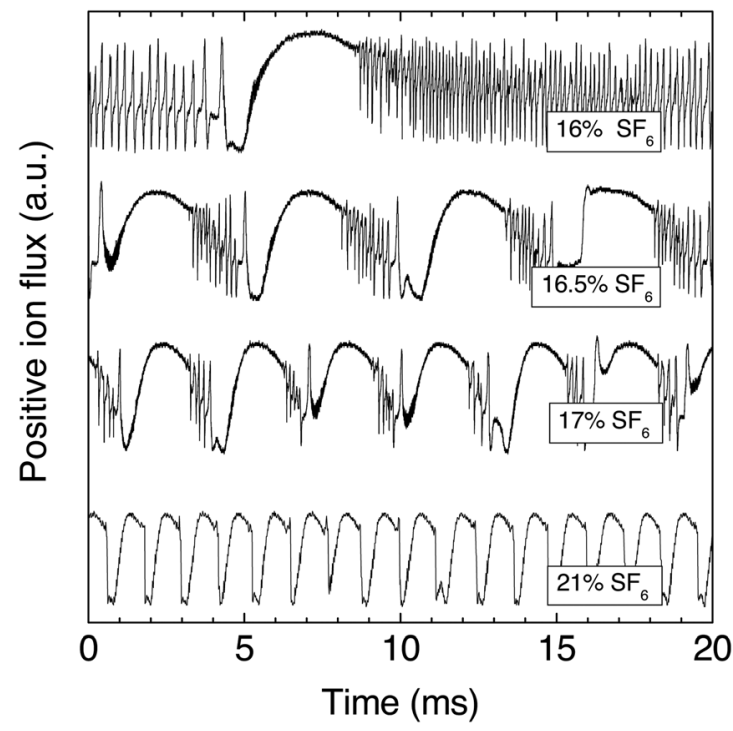

FIG. 8. Temporal evolution of the positive ion saturation current for increasing $\mathrm{SF}_{6}$ concentration. As the $\mathrm{SF}_{6}$ concentration is increased, the typical time for "negative ion fraction increase" decreases and ion acoustic fluctuations quickly reach the non-linear regime (above $21 \%$, the ion acoustic fluctuations are no longer observed). 
evolution of the ion acoustic fluctuations) is shorter than the most unstable ion acoustic wave period. The transition from Regime V to Regime VI is, thus, driven by the ratio $\tau$ of the most unstable ion acoustic wave period over the negative-ion time-scale: Regime $\mathrm{V}$ is observed for $\tau<2$, while Regime VI is observed for $\tau>2$. The operational conditions of Tuszewski and coworkers ${ }^{6,7}$ are similar to the one of Regime VI (namely, they used equal $\mathrm{SF}_{6}$ and Argon partial pressures), and it is likely that, similarly to our observations, they indeed observed non-linearly unstable ion acoustic waves, and possibly double layer formation and propagation.

\section{CONCLUSIONS}

Unsteady plasma operations in electronegative gases have long been observed. Tuszewski and coworkers showed that, in an elongated plasma vessel, a transport instability was likely to occur. ${ }^{5,6}$ They analyzed the instability as a nonlinear evolution of ion acoustic waves, driven unstable by oppositely streaming positive and negative ions. ${ }^{6}$ We later showed that, in similar operational conditions, a transport instability was caused by the pseudo-periodic formation and propagation of a moderate amplitude double layer. ${ }^{8}$ For very low negative ion fraction, the double layer was static and no plasma parameter fluctuations were observed. ${ }^{9}$ We showed in this paper, that beside the existence of these double layers, slow ion acoustic waves could be driven unstable in expanding electronegative plasmas, as analyzed by Tuszewski and Gary. ${ }^{6}$ These unstable ion acoustic waves propagate upstream, non-linearly interact and show turbulent features. We also showed that these unstable ion acoustic waves are linked with double layer formation in electronegative plasmas.

These scenarios were analyzed in $\mathrm{Ar} / \mathrm{SF}_{6}$ gas mixture, by increasing gradually the $\mathrm{SF}_{6}$ concentration, i.e., gradually increasing the negative ion fraction. The following sequence was thus determined:

- I-For very low negative ion fractions, the plasma continuously expands in a steady state equilibrium

- II-For negative ion fraction around unity, ion acoustic waves are driven unstable in the expansion region, and propagate toward the source region.

- III-For moderate negative ion fractions, the ion acoustic waves are driven non-linearly unstable, and a double layer forms at the expansion region. This double layer may be static at moderate negative ion fraction (around 3).

- IV-In the presence of a static double layer, ion acoustic waves could then be driven unstable in the downstream region.
- $\mathrm{V}$-For high negative ion fractions, ion acoustic waves are quickly driven non-linearly unstable, and double layer periodically form and propagate downstream.

These observations corroborate previous numerical simulations were static double layers were observed at moderate negative ion fraction (and low pressure) ${ }^{13,14}$ and propagative double layers at high negative ion fractions. ${ }^{13}$ As Kawamura and coworkers, ${ }^{15}$ we also observed ion acoustic waves in the downstream region of a static double layer. Nevertheless, contrary to the numerical simulations, where the range of instability for the ion acoustic waves is narrower than the range of parameters were double layers are observed, we found that unstable ion acoustic waves are more generic than double layers. Moreover, our experimental results suggest that, in electronegative plasmas, double layer formation is the non-linear evolution of unstable, large amplitude ion acoustic waves.

${ }^{1}$ M. A. Lieberman and A. J. Lichtenberg, Principles of Plasma Discharges and Materials Processing, 2nd ed. (Wiley-Interscience, USA, 2005).

${ }^{2}$ E. Metsi, E. Gogolides, and A. Boudouvis, Phys. Rev. E 54, 782 (1996).

${ }^{3}$ P. Chabert, A. J. Lichtenberg, M. Lieberman, and A. M. Marakhtanov, Plasma Sources Sci. Technol. 10, 478 (2001).

${ }^{4}$ P. Chabert, A. Lichtenberg, M. Lieberman, and A. Marakhtanov, J. Appl. Phys. 94, 831 (2003)

${ }^{5}$ M. Tuszewski, J. Appl. Phys. 79, 8967 (1996).

${ }^{6}$ M. Tuszewski and R. R. White, J. Appl. Phys. 94, 2858 (2003).

${ }^{7}$ M. Tuszewski and S. P. Gary, Phys. Plasmas 10, 539 (2003).

${ }^{8}$ N. Plihon, C. S. Corr, P. Chabert, and J.-L. Raimbault, J. Appl. Phys. 98, 023306 (2005).

${ }^{9}$ N. Plihon, C. S. Corr, and P. Chabert, Appl. Phys. Lett. 86, 091501 (2005).

${ }^{10}$ C. Hayden, D. Gahan, and M. B. Hopkins, Plasma Sources Sci. Technol. 18, 025018 (2009)

${ }^{11}$ N. Plihon, P. Chabert, and C. S. Corr, Phys. Plasmas 14, 013506 (2007).

${ }^{12}$ N. Plihon, Ph. D.thesis, École Polytechnique, France - http://tel.ccsd. cnrs.fr/tel-00083948, 2006.

${ }^{13}$ A. Meige, N. Plihon, G. J. M. Hagelaar, J.-P. Boeuf, P. Chabert, and R. W. Boswell, Phys. Plasmas 14, 053508 (2007).

${ }^{14}$ E. Kawamura, A. J. Lichtenberg, M. A. Lieberman, and J. P. Verboncoeur, Phys. Plasmas 16, 122114 (2009).

${ }^{15}$ E. Kawamura, A. J. Lichtenberg, and M. A. Lieberman, J. Appl. Phys. 107, 123301 (2010).

${ }^{16}$ R. W. Boswell, Phys. Lett. 33A , 457 (1970).

${ }^{17}$ A. Cantin and R. R. J. Gagne, Appl. Phys. Lett. 30, 316 (1977)

${ }^{18}$ M. B. Hopkins and W. G. Graham, Rev. Sci. Instr. 57, 2210 (1986).

${ }^{19}$ F. Magnus and J. T. Gudmundsson, Rev. Sci. Instrum. 79, 073503 (2008).

${ }^{20} \mathrm{P}$. Chabert, T. Sheridan, R. Boswell, and J. Perrin, Plasma Sources Sci. Technol. 8, 561 (1999)

${ }^{21}$ M. Bacal, Rev. Sci. Instr. 71, 3981 (2000).

${ }^{22}$ W. D. Jones, H. J. Doucet, and J. M. Buzzi, An Introduction to the Linear Theories Ans Methods of Electrostatic Waves In Plasmas (Plenum, New York, 1985). 